\title{
A left-lateralized network for reading Chinese words: a 3 T fMRI study
}

\author{
Wen-Jui Kuo, ${ }^{1,2}$ Tzu-Chen Yeh, ${ }^{1,3}$ Jeng-Ren Duann, ${ }^{1,4}$ Yu-Te Wu, ${ }^{1,5}$ Low-Tone Ho, ${ }^{1,3}$ Daisy Hung, ${ }^{2,6}$ \\ Ovid J. L. Tzeng ${ }^{2,6}$ and Jen-Chuen Hsieh ${ }^{1,3,6, C A}$
}

\begin{abstract}
'Integrated Brain Research Laboratory, Department of Medical Research and Education, Taipei Veterans General Hospital, No.20I, Sect.2, Shih-Pai Rd., Taipei I I 2; ${ }^{2}$ Cognitive Neuropsychology Laboratory, ${ }^{3}$ Departments of Anesthesiology, Psychiatry, and Radiology, Faculty of Medicine, School of Medicine, ${ }^{5}$ Institute of Radiological Sciences, and ${ }^{6}$ Institute of Neuroscience, School of Life Science, National Yang-Ming University Taipei, Taiwan; ${ }^{4}$ Computational Neurobiology Laboratory, Salk Institute, USA
\end{abstract}

CA,I Corresponding Author and Address

Received 28 September 200I; accepted 15 October 200 I

fMRI was used to investigate brain organization for reading in Chinese. Subjects were shown two-character Chinese words. A control task was used to eliminate the non-linguistic visual and motor confounds. Results show that naming of Chinese logographs is characterized by left-lateralized neuronal networks for the processing of orthographic, phonological, and semantic attributes. The orchestration of the middle frontal cortex, superior temporal cortex, superior parietal cortex, basal temporal area and extrastriate cortices of the left hemisphere may manifest the particularity of the central representation of simple word naming in Chinese. NeuroReport |2:3997-400 I (C) 200 I Lippincott Williams \& Wilkins.

Key words: Brain; Chinese; fMRI; Reading; Word recognition

\section{INTRODUCTION}

Word recognition in reading consists of many processes that collectively activate several specialized neural systems to work in concert. In the proposed word recognition model [1], reading printed words may on one hand target the posterior fusiform and lingual gyri for visual processing while engaging the left posterior middle temporal, posterior temporoparietal and anterior inferior temporal cortices for semantic processing. However, there are many differences in the orthographic structure of different languages. The extent to which orthographic variations of different languages can influence reading processes in the brain has not been well elucidated.

The surface form of a writing system can influence the reading process and its cerebral organization [2,3]. The idea that specific processing requirements of the languages may forge the organization of the language systems of the brain has gained support from brain imaging research on American Sign Language (ASL) showing an engagement of the right hemisphere in addition to the left hemispheric structures classically linked to the processing of spoken language [3]. Behavioral studies also indicate that differences in script have an impact on visual information processing during reading, such as visual scanning and perceptual demands [4].

Chinese logographs differ markedly from languages represented using an alphabet in phonology and orthography, as there is no eminent letter-sound correspondence. Written Chinese uses characters as basic units of written language. These characters have a square configuration of similar size, and map onto morphemes (meaning) rather than phonemes in the spoken language [5]. Each Chinese character contains radicals, or sub-lexical structural units (including strokes, dots and curves; Fig. 1a), which might hint at either the pronunciation or the semantics of the character. These unique characteristics imply that some of the neuropsychological mechanisms underlying the reading of Chinese logographs may differ from those used in reading alphabetic words.

The idea that a logographic system mandates elaborative visuospatial processing is corroborated by behavioral [2] and brain imaging studies [5,6]. Tan et al. report an extensive activation of bilateral hemispheric structures in Chinese character processing, using a semantic judgment task and a homophone judgment task $[5,6]$. Of particular interest is activation in the left middle frontal gyrus (BA 9), an area pertinent to spatial working memory [7], was consistently found across the studies and is distinct from studies on alphabetic languages. The authors suggest that the left middle frontal area coordinates and integrates visuospatial analysis demanded by the logographs square configuration and the semantic (or phonological) analysis $[5,6,8]$.

However, findings from brain imaging studies on Chinese characters are at odds with studies on Japanese Kanji. Japanese Kanji retain the orthographic architecture of Chinese characters, and each represents a lexical morpheme of spoken Japanese. Studies on brain lesions suggest that the processing of Kana (a phonetic-syllabic system used in writing Japanese) targets the dorsal stream 


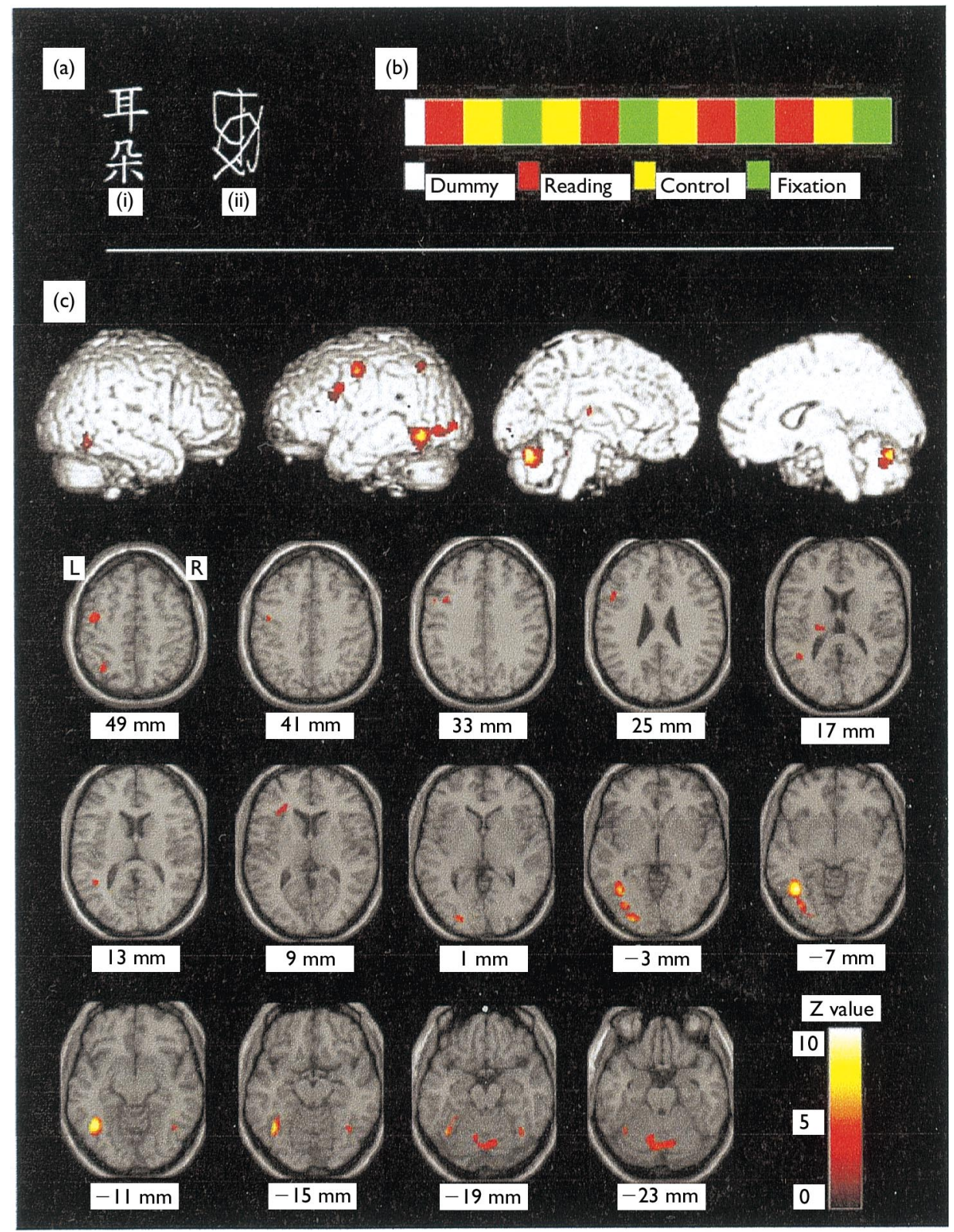

Fig. I. Experimental protocol and functional maps. (a) An example of the word stimuli. (i) An example of Chinese word (meaning ear in this case) used in the experiment. (ii) An example of nonsense figures. (b) Experimental paradigm. (c) Statistical maps of reading vs. control. Voxels displayed survive the uncorrected threshold of $p=0.0001$. The color bar denotes $Z$ max values.

(from the angular gyrus to Wernicke's area), whereas the processing of Kanji (the Japanese logographic system) mainly engages the ventral route (from the posterior inferior temporo-occipital area, PITOA, to Wernicke's area) of the left hemisphere $[9,10]$. This theory is supported by brain imaging studies [11-13]. Furthermore, using wellcontrolled tasks of Kana-to-Kanji transcription and mental recall of Kanji orthography, Nakamura et al. [14] report a left-lateralized activation in the PITOA. Neither oral reading nor semantic judgment tasks involving words written in Kana activated this area [14]. Converging evidence shows that the left PITOA plays a critical role in processing for writing Kanji, mediating the retrieval of the visual graphic image.

Surprisingly, the activation of the PITOA has not been consistently reported in imaging studies on Chinese character processing $[5,6,8]$. We reason that the lack of consistent activation of the PITOA in Tan's series of imaging studies could be ascribed in part to different task demands (e.g. word generation) in the studies $[5,6,8]$. Furthermore, using the fixation condition as a common baseline in these studies, the caveat exists that neither the non-linguistic processes of visual input nor those of motor output were well controlled. Using scrambled Kanji as a strict percep- 
tual control for naming Kanji covertly, Uchida et al. [13] report that the left PIOTA is prominently activated in orthographic processing of Japanese kanji.

The present study utilizes whole-brain $3 \mathrm{~T}$ fMRI to observe brain response during the reading of Chinese words. The goals of the current study are threefold. First, the study inspects the commonality and particularity of brain organization for reading Chinese relative to alphabetic languages. Second, the study exploits a visuomotor control task to eliminate the non-linguistic visual and motor confounds in order to reconcile the discrepancies between studies on Japanese Kanji and Chinese characters/words. Third, the study proposes that Chinese word recognition might mandate perceptual and attentional mechanisms that target the left hemisphere, advantageous for local details, for the fine-grained analysis of spatial properties of Chinese characters.

\section{MATERIALS AND METHODS}

Subjects: Seven right-handed university students (four males), with a mean age of 24 years (range 21-27) were recruited for the study. All volunteers were native Chinese speakers without any history of neurological disorders, and had normal or corrected to normal vision. Handedness of the subjects was verified using the Edinburgh Inventory [15]. Consent for the study was obtained from all participants with the protocol approved by the Institutional Ethics and Radiation Safety Committees.

Experimental design: Three blocked conditions were implemented. In the reading task (R), subjects read the displayed two-character words covertly (Fig. 1ai). In the control task (C), meaningless figures were presented. Subjects were asked to pronounce /dida/ covertly for each figure displayed. The stimuli served as a control for Reading to remove non-linguistic components of visual input and motor output (Fig. 1aii). In the fixation condition (F), serving as the common baseline, subjects were requested to fixate on the crosshair presented, with no response required.

The two-character words used in the present study were concrete nouns with frequency counts ranging from middle to high level [16]. The stimuli were presented for $1 \mathrm{~s}$, alternating with a blank field of $1 \mathrm{~s}$ duration. Each condition persisted for $20 \mathrm{~s}$. The presentation order (e.g., RCFCRFCRFRCF) was balanced within and across the subjects (Fig. 1b). Stimuli were presented with a PC using a custom-designed program and projected via an LCD projector (Toshiba TY-G3, Japan) outside the shielded room on a screen at the feet of the subject. Viewing distance was about $194 \mathrm{~cm}$. Subjects viewed the stimuli via a homemade reflection mirror. The visual angle of each Chinese character and the control figure subtended $\sim 2-3^{\circ}$, while that of the fixation crosshair subtended $\sim 1^{\circ}$.

fMRI scanning: Images were acquired using a $3.0 \mathrm{~T}$ Bruker MedSpec S300 system (Bruker, Kalsrube, Germany) with a quadrature head coil. Subjects' heads were immobilized with a vacuum-beam pad in the scanner. Functional data were acquired with a T2*-weighted gradient-echo EPI using BOLD contrast $\left(\mathrm{TR} / \mathrm{TE} / \theta=2000 \mathrm{~ms} / 50 \mathrm{~ms} / 90^{\circ}\right.$, slice thickness $=5 \mathrm{~mm}$, inter-slice interval $=1 \mathrm{~mm}$, FOV $=$
$250 \mathrm{~mm}, 64 \times 64 \times 20$ matrix, whole brain covered). For each slice, 240 images were acquired. The first five images (dummy image) were discarded from the analysis to eliminate non-equilibrium effects of magnetization (Fig. 1b). The anatomical image was acquired using a high-resolution T1weighted, 3D gradient-echo pulse sequence (MDEFT: Modified Driven Equilibrium Fourier Transform; TR/TE/TI = $88.1 \mathrm{~ms} / 4.12 \mathrm{~ms} / 650 \mathrm{~ms}, 128 \times 128 \times 128$ matrix, $\mathrm{FOV}=$ $250 \mathrm{~mm})$.

fMRI analysis: Data were analyzed, with statistical parametric mapping (SPM99 software from the Welcome Department of Cognitive Neurology, London), running under Matlab 6.0 (Mathworks, Sherbon, MA, USA). The first five images (dummy image) were discarded from the analysis to eliminate non-equilibrium effects of magnetization (Fig. 1b). Scans were realigned, normalized, and spatially smoothed using an $8 \mathrm{~mm}$ FWHM isotropic Gaussian kernel. The resulting time series data across sessions were high-pass filtered with a cut-off of $120 \mathrm{~s}$ to remove low frequency drift, and temporally smoothed using a hemodynamic response function. Contrasts between conditions (reading vs fixation and reading $v s$ control were examined by voxel-specific t-tests $\{\mathrm{SPM}(\mathrm{t})\}$ across all participants. The t-statistics were subsequently transformed to $\mathrm{Z}$ statistics to create a statistical parametric map $\{\operatorname{SPM}(\mathrm{z})\}$ for each contrast. The regionally specific differences with an uncorrected conservative threshold of $p=0.0001$ ( $Z=3.72$, with the cluster size $>10$ voxels) were considered statistically significant. Maxima were localized on the normalized structural images and 1abeled using the nomenclature of Talairach. The contrast of reading us control yields a brain activation pattern for Chinese word reading.

\section{RESULTS}

Reading vs fixation: Activation of the bilateral precentral gyri, left medial and inferior frontal gyri, right middle frontal gyras, and bilateral cingulate gyri were observed (Table 1). Parietal activation was seen in the left postcentral gyrus, bilateral superior parietal lobule, and right precuneus. Temporal activation was observed in the left superior temporal and right middle temporal gyri. Reading also activated the bilateral cuneus, lingual, and inferior/middle occipital gyri as well as the fusiform gyri. Activation of the left thalamus, right pulvinar, bilateral cerebellum and lentiform nuclei was also noted.

Reading vs control: Reading, referenced to visuomotor control, activated the precentral, middle frontal, and inferior frontal gyri of the left hemisphere (Table 1, Fig. 1c). Parietal activation was seen in the left superior parietal lobule. In the temporal cortex, activation was found in the left superior temporal gyrus. Reading activated the bilateral fusiform, left middle occipital gyrus, and left inferior occipital gyrus. Activation of the left insula, left thalamus, and bilateral cerebellar hemispheres was also noted.

\section{DISCUSSION}

The present study aimed to investigate the commonality and particularity of brain organization for reading the logographic Chinese and other, alphabetic, languages. Two-character words were chosen as stimuli since such 
Table I. Foci of activation during reading.

\begin{tabular}{|c|c|c|c|c|c|c|c|c|c|c|}
\hline \multirow[t]{2}{*}{ Brain region } & \multicolumn{5}{|c|}{ Left hemisphere } & \multicolumn{5}{|c|}{ Right hemisphere } \\
\hline & BArea & $x$ & y & z & $Z$ value & BArea & $x$ & y & z & $Z$ value \\
\hline \multicolumn{11}{|l|}{ Reading vs fixation } \\
\hline \multicolumn{11}{|l|}{ Frontal lobe } \\
\hline Medial frontal gyrus & 6 & -6 & 0 & 60 & 5.01 & & & & & \\
\hline Precentral gyrus & 6 & -44 & -2 & 48 & infinity & 6 & 58 & -2 & 32 & 6.39 \\
\hline Inferior frontal gyrus & 9 & -52 & 12 & 30 & 5.89 & 6 & 52 & 0 & 46 & 5.48 \\
\hline Middle frontal gyrus & & & & & & 6 & 38 & -6 & 44 & 5.40 \\
\hline Cingulate gyrus & 24 & -10 & -8 & 26 & 5.06 & 24 & 10 & -6 & 28 & 5.39 \\
\hline \multicolumn{11}{|l|}{ Parietal lobe } \\
\hline \multirow[t]{2}{*}{ Postcentral gyrus } & 43 & -60 & -6 & 20 & 5.50 & & & & & \\
\hline & 2 & -50 & -20 & 42 & 5.45 & & & & & \\
\hline Superior parietal lobule & 7 & -28 & -50 & 50 & 5.69 & 7 & 36 & -64 & 34 & 4.72 \\
\hline Precuneus & & & & & & 19 & 32 & -58 & 38 & 4.71 \\
\hline \multicolumn{11}{|l|}{ Temporal lobe } \\
\hline Superior temporal gyrus & 22 & -44 & -48 & 16 & 3.91 & & & & & \\
\hline Middle temporal gyrus & & & & & & 21 & 54 & -34 & 0 & 4.33 \\
\hline \multicolumn{11}{|l|}{ Occipital lobe } \\
\hline Fusiform gyrus & 37 & -38 & -56 & -6 & infinity & 37 & 36 & -50 & -12 & infinity \\
\hline Cuneus & 17 & -12 & -92 & 4 & infinity & 18 & 22 & -86 & 8 & infinity \\
\hline Lingual gyrus & 18 & -18 & -82 & -2 & infinity & 18 & 34 & -72 & -8 & infinity \\
\hline \multirow[t]{2}{*}{ Middle occipital gyrus } & 18 & -16 & -88 & 14 & infinity & 37 & 38 & -62 & 0 & infinity \\
\hline & 19 & -26 & -86 & 6 & infinity & 19 & 30 & -84 & 12 & infinity \\
\hline \multicolumn{11}{|l|}{ Thalamus } \\
\hline & & -24 & -28 & 2 & 7.09 & & & & & \\
\hline Lentiform nucleus & & -20 & -16 & 14 & 6.12 & & 24 & -10 & 4 & 5.90 \\
\hline Pulvinar & & -28 & -12 & -4 & 5.50 & & 24 & -28 & 4 & 4.54 \\
\hline Cerebellum & & -8 & -62 & -12 & 6.24 & & 34 & -58 & -16 & infinity \\
\hline \multicolumn{11}{|l|}{ Reading vs control } \\
\hline \multicolumn{11}{|l|}{ Frontal lobe } \\
\hline Precentral gyrus & 6 & -44 & -4 & 44 & 5.70 & & & & & \\
\hline Middle frontal gyrus & 9 & -40 & 12 & 28 & 4.42 & & & & & \\
\hline Inferior frontal gyrus & 9 & -48 & 14 & 24 & 4.54 & & & & & \\
\hline Insula & 13 & -34 & 26 & 10 & 3.96 & & & & & \\
\hline \multicolumn{11}{|l|}{ Parietal lobe } \\
\hline Superior parietal lobule & 7 & -34 & -56 & 48 & 4.17 & & & & & \\
\hline \multicolumn{11}{|l|}{ Temporal lobe } \\
\hline Superior temporal gyrus & 22 & -42 & -48 & 16 & 4.54 & & & & & \\
\hline \multicolumn{11}{|l|}{ Occipitotemporal region } \\
\hline Inferior temporal gyrus & 37 & -40 & -54 & -2 & infinity & & & & & \\
\hline Middle occipital gyrus & 18 & -26 & -88 & 2 & 6.75 & & & & & \\
\hline Inferior occipital gyrus & 19 & -34 & -72 & 0 & 6.32 & & & & & \\
\hline Fusiform gyrus & 37 & -36 & -46 & -12 & 4.91 & 37 & 40 & -56 & -10 & 5.19 \\
\hline Thalamus & & -22 & -18 & 16 & 4.20 & & & & & \\
\hline \multirow[t]{2}{*}{ Cerebellum } & & -2 & -72 & -14 & 5.11 & & 10 & -70 & -14 & 5.30 \\
\hline & & & & & & & 2 & -66 & -20 & 4.48 \\
\hline
\end{tabular}

Infinity: $Z$ value $>8$; BArea $=$ Brodmann's Area.

entities comprise almost $50 \%$ of the Chinese vocabulary [16]. In contrast to visuomotor control, results support a left-hemispheric advantage in orchestrating orthographic, phonological, and semantic processing for reading Chinese words.

The orthographical aspect of lexical processing is the most fundamental stage of visual word recognition. We observed a left-lateralized activation of extrastriate areas (lateral inferior/middle occipital gyrus; areas 18 and 19), basal temporal cortex (area 37, bilaterally activated but left hemisphere dominant), and superior temporal region (area 22 ) in the contrast of reading vs control (Table 1, Fig. 1c). Our data support the findings of Uchida et al. concerning the processing of Japanese Kanji [13], and are congruent with the results from other studies on the processing of logographs [11,17] and alphabetic scripts [1,18,19], respectively. These observations are in line with theories asserting that these regions service orthographic processing and lexical retrieval, interfacing between the perception and the long-term mental representation of words [1,18,19].

The left-lateralized activation of the anterior insula can be better appreciated under the context of concomitant activation of the left premotor cortex (area 6). The left insula and left premotor cortex work in concert in formulating articulatory plans and coordinating speech articulation $[1,20,21]$. The lack of activation in Broca's area (frontal operculum and posterior third of the inferior frontal gyrus) is congruent with results in studies on alphabetic lan- 
guages and supports the idea that Broca's area may be engaged in tasks commanding more subtle and complex computations, such as syntactic or grammatical analysis $[21,22]$.

Some activated regions had not been identified as critically linked to reading in previous studies on alphabetic languages. After eliminating the contribution from the non-linguistic visuospatial and motor components, a left-lateralized activation of dorsal middle/inferior frontal areas was observed. The left middle/inferior frontal gyri (area 9) have been demonstrated to mediate spatial and verbal working memory [7] and may subserve a central executive function inherent for the coordination of cognitive resources [23]. These regions have been proposed as coordinating and integrating the intensive visuospatial analyses of Chinese logographs and phonological computation, as well as semantic analysis [5,6].

The activation pattern in the reading $v$ s fixation contrast are generally similar to the findings reported by Tan et al. $[5,6]$. However, prominent differences were noted when contrasting the reading condition with visuomotor control. Contrary to results in previous fMRI studies on reading of Chinese characters using sophisticated tasks of word generation and semantic/homophone decision (with a crosshair fixation condition as the common control), no significant activity in the regions of right hemisphere, e.g. superior frontal, parietal, and precuneus, was detected in the contrast of reading vs control. The aforementioned regions have been suggested to specifically take part in the visuospatial analyses of Chinese character $[5,6,8]$. Based on the left-lateralized concomitant activation of the middle/ inferior frontal and superior parietal cortices, we speculate that recognition of Chinese characters/words may elaborate more perceptual and attentional mechanisms than recognition of alphabetic scripts, due to perception of the spatial locations of the strokes and the architecture of the stroke combinations in a square. This may target the neural networks of the left hemisphere, which has an advantage for local details [24,25], for the fine-grained analysis of spatial properties of Chinese characters.

\section{CONCLUSION}

Different languages may shape different brain organizations for their processing. Using a visuomotor control task to eliminate the non-linguistic visual and motor confounds, we have shown that naming of Chinese logographs is characterized by left-lateralized neural networks for the processing of orthographic, phonological, and semantic attributes. The brain activation, comprising concomitant activation of middle frontal cortex, superior temporal cortex, superior parietal cortex, basal temporal area and extrastriate cortices of the left hemisphere, may succinctly manifest the particularity of the central representation of simple word naming in Chinese.

\section{REFERENCES}

1. Price CJ. J Anat 197, 335-359 (2000)

2. Tzeng OJL and Wang WSY. Am Sci 71, 238-243 (1983).

3. Neville HJ, Bavelier D, Corina D et al. Proc Natl Acad Sci USA 95, 922-929 (1998).

4. Hung DL and Tzeng OJL. Psychol Bull 90, 377-414 (1981).

5. Tan LH, Liu HL, Perfetti CA et al. NeuroImage 13, 836-846 (2001).

6. Tan LH, Feng CM, T Fox PT and Gao JH. Neuroreport 12, 83-88 (2001).

7. Courtney SM, Petit L, Maisog JM et al. Science 279, 1347-1351 (1998).

8. Tan LH, Spinks JA, Gao JH et al. Hum Brain Mapp 10, 16-27 (2000).

9. Iwata M. Trends Neurosci 7, 290-293 (1984).

10. Soma Y, Sugishita M, Kitamura Y et al. Brain 112, 1549-1561 (1989).

11. Sakurai Y, Momose T, Iwata M et al. Cogn Brain Res 9, 111-115 (2000).

12. Law I, Kannao I, Fujita H et al. J Neurolinguistics 6, 243-251 (1991).

13. Uchida I, Kikyo H, Hakajima K et al. Neuroimage 9, 208-215 (1999).

14. Nakamura K, Honda M, Okada T et al. Brain 123, 954-967 (2000).

15. Oldfield RC. Neuropsychologia 9, 97-113 (1971).

16. Academia-Sinica. Academia Sinica Balanced Corpus (electronic database), 3rd edn. Taipei: Academia Sinica; 1998.

17. Chee MW, Tan E and Thiel T. J Neurosci 19, 3050-3056 (1999).

18. Moore CJ and Price CJ. Neuroimage 10, 181-192 (1999).

19. Wise RJS, Scott SK, Blank SC et al. Brain 124, 83-95 (2001).

20. Dronkers NF. Nature 384, 159-161 (1996).

21. Wise RJS, Greene J, Buchel C and Scott SK. Lancet 353, 1057-1061 (1999).

22. Petersen SE, Fox PT, Posner MI et al. Nature 331, 585-589 (1988).

23. D'Esposito M, Detre JA, Alsop DC et al. Nature 378, 279-281 (1995).

24. Fink GR, Halligan PW, Marshall JC et al. Nature 382, 626-628 (1996).

25. Robertson LC, Lamb MR and Knight RT. J Neurosci 8, 3757-3769 (1988).

Acknowledgements: Supported by grants from the Taipei Veterans General Hospital (874l4, 88377, 89362, 89402), National Science Council (9025I I SOI000I), Academia Sinica (87, 88), and Ministry of Education (89BFA22I40I) of Taiwan. Special thanks to $\mathrm{Mr}$ Chi-Cher Chou for MRI technical assistance. 